EXPERTMENTAL NEUROLOGY 6, 478-491 (1962)

\title{
Surgical Interference with Pathways Mediating Responses Conditioned to Cortical Stimulation
}

\author{
L. T. Rutledge AND R. W. Doty ${ }^{1}$ \\ Department of Physiology, University of Michigan Medical School, \\ Ann Arbor, Michigan
}

Received July 30, 1962

Cats trained to avoid a foot shock (US) when electrical stimulation (CS) was applied to homotopic areas on either the right or left marginal gyrus were subjected to various degrees of surgical isolation of the stimulated areas. For ten cats a U-shaped incision on one hemisphere isolated the stimulated area from most of the intracortical connections, and the contralateral area was undercut to eliminate direct corticifugal conduction. Postoperative tests showed a highly significant superiority in conditioned reflex (CR) lcvel for stimulation of U-cut cortex. Cats with cortex undercut had to be retrained before a stable level of CR could be reestablished. Four other animals underwent bilateral undercutting of varying extents so that the reorganization could be more carefully evaluated. Trigeminal neurotomies and other controls were utilized. It is concluded that the electrically applied CS normally is elaborated principally via corticifugal fibers and if these fibers are cut, additional training is necessary before the intracortically conducted CS can become effective.

\section{Introduction}

In the endeavor to understand conditioned reflexes (CR) in terms of neural processes, one of the major difficulties is the inability to specify which neurons participate in the phenomenon. By applying the conditional stimulus (CS) directly to the central nervous system it can be shown $(6,7,9)$ that the sensory systems normally utilized are at least dispensable. It is thus possible to examine and surgically modify various central pathways to study their capacity for mediating an effective CS and,

1 Aided by grants B-663 and B-1068 from the National Institute of Neurological Diseases and Blindness, and Senior Research Fellowship GM-K3-15, 122 (Rutledge) from the U. S. Public Health Service. Dr. Doty's present address is: Center for Brain Research, University of Rochester, Rochester, New York. 
ultimately, to determine what distinguishes the effective from the ineffective pathway.

For an electrical CS applied to the cerebral cortex, the present data support those in our progress reports $(20,8)$ and show that corticifugal, and not intracortical, paths initially mediate the CS. However, after elimination of corticifugal fibers, effective intracortical paths can be established with retraining.

\section{Method}

The fourteen adult cats on which the data were obtained were successfully carried through most stages of the experiment. Others died after surgery or developed neurological complications which rendered them unfit for continued experimentation. Either before initial training (three) or after cortical incisions and retraining, seven of the fourteen had bilateral trigeminal neurotomies and two others were unilaterally operated. A direct surgical procedure (6) was followed for the neurotomies, which were performed under deep barbiturate anesthesia and sterile conditions. The adequacy of the surgery was evaluated by the absence of the corneal reflex, and confirmed at necropsy.

The training technique previously described (7) was used. This consisted of applying a 2-sec electrical stimulation (1-msec pulses, 50/sec) to the cerebral cortex to serve as CS and pairing this with shock to the right foreleg as the unconditional stimulus (US). Each animal was trained to respond at a CR level of 60 to $100 \%$ to stimulation of homotopic right or left marginal gyrus loci via two sets of electrodes. Most animals were also trained to respond to an auditory or photic CS and the CR elicited by such peripheral stimuli provided a valuable indication of the animal's general condition following surgical procedures.

After training was complete the cortex underlying the implanted electrodes was subjected to various surgical isolations. On one side corticifugal fibers from the CS area were severed by undercutting the stimulated cortex at a depth of 3 to $5 \mathrm{~mm}$, to a midline falx cerebri, and to a length usually of 9 to $15 \mathrm{~mm}$. The procedure is diagrammed in Fig. 1 and histological sections shown in Figs. 2, 3, 4 and 5. Five of the fourteen animals underwent bilateral undercuts of varying extents.

At the contralaterally implanted area, the electrode button was removed and the area debrided of connective tissue, bone, and dural regrowth. The cortex was incised to a depth of 4 or $5 \mathrm{~mm}$ with a U-shaped cut at least $8 \mathrm{~mm}$ in diameter (Figs. 1-6). The shape of this cut was dictated 
by the necessity of keeping the pial 'circulation intact (23). That pial circulation of a $U$-shaped cut of this type is adequate to maintain viable tissue was shown in three cats (not in the present series) in which the postoperative circulatory pattern was studied. As shown in Fig. 6, a full

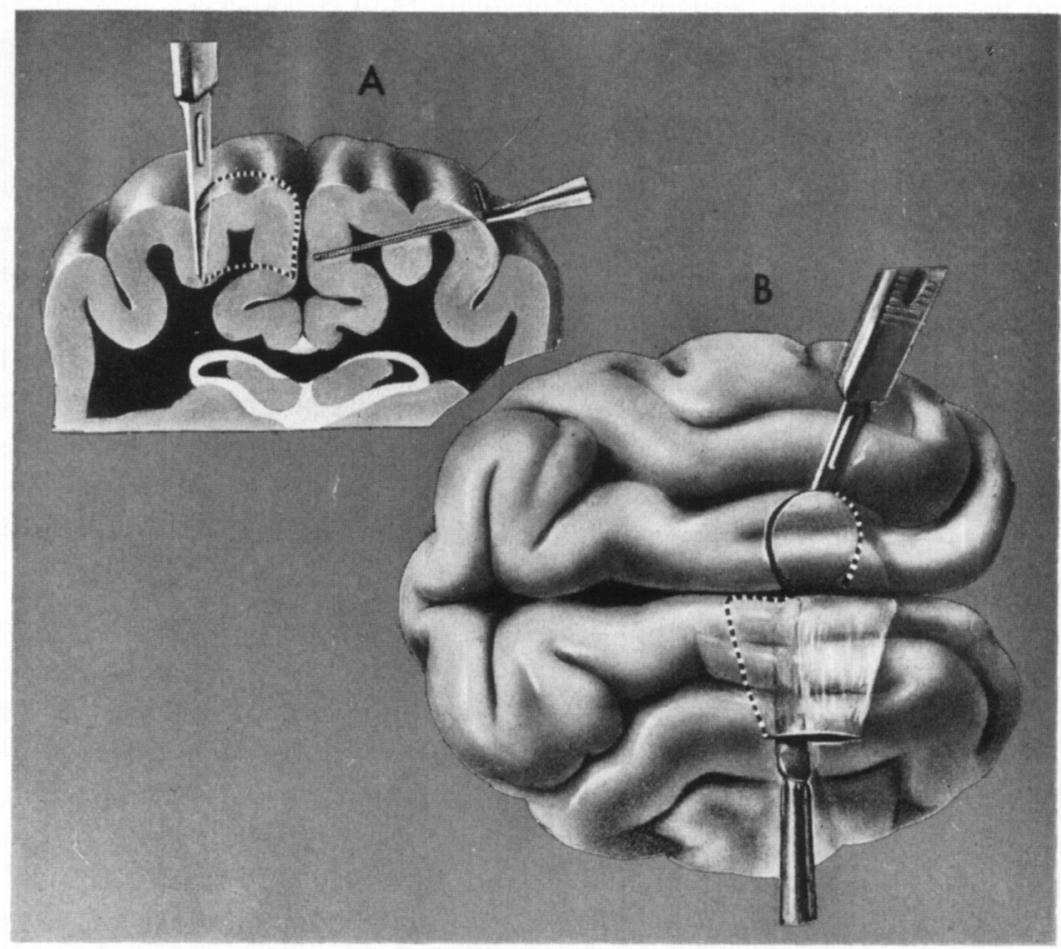

FIg. 1. Surgical technique for partial isolation of cortical areas. Anterior is to the left on the dorsal view, B. The U-shaped cut was made with the knife held vertically, except as the midline was approached the knife was angled so as to cut close to the falx cerebri and subpially at the surface. As viewed from $B$, the medial portion of the $U$ contained intact intracortical fibers coextensive with cortex just dorsal to the splenial sulcus. Undercutting was always to the midline and usually angled as shown in $\mathrm{A}$.

circumsection of cortex and pia mater leads to degeneration whereas the U-shaped cut does not. In the case of the U-shaped cut blood vessels proliferate and even grow across the scar (Fig. 6). Histology also verified the occurrence of vascularization across the scar in the depths of the isolated tissue. 
After postsurgical recovery the animals usually received auditory CS presentations as a control on CR level. They were then tested with central CS presentations to determine the relative effectiveness of the CS when applied to the undercut and U-cut cortex. Retraining was continued to a
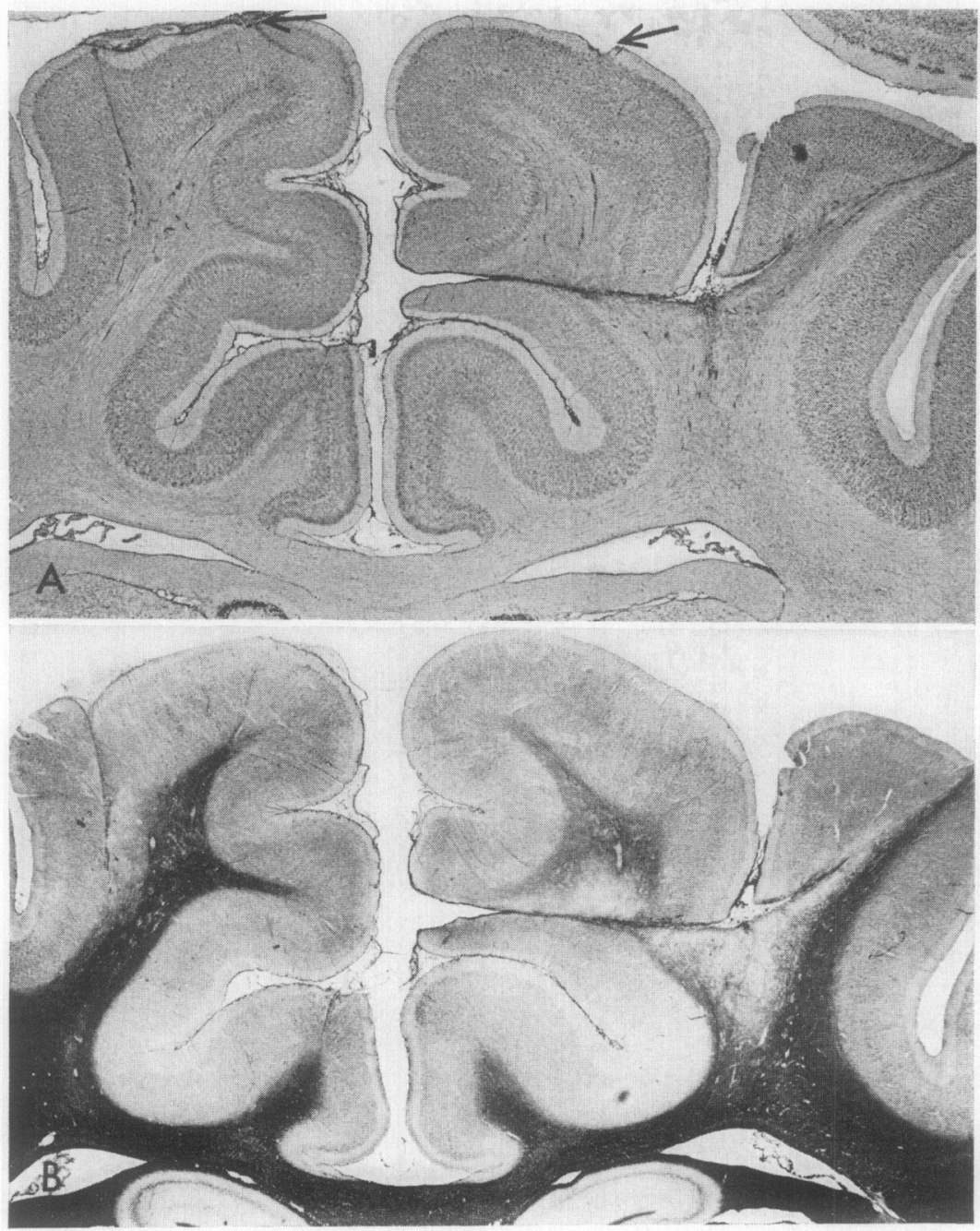

FIr. 2. Histological sections of partially isolated cortex; cat 58-6, killed 3 months after cortical "isolations." Arrows point to two electrode locations. Discussion of performance in text. A, Nissl; B, Weil stain; $8 \times$. 

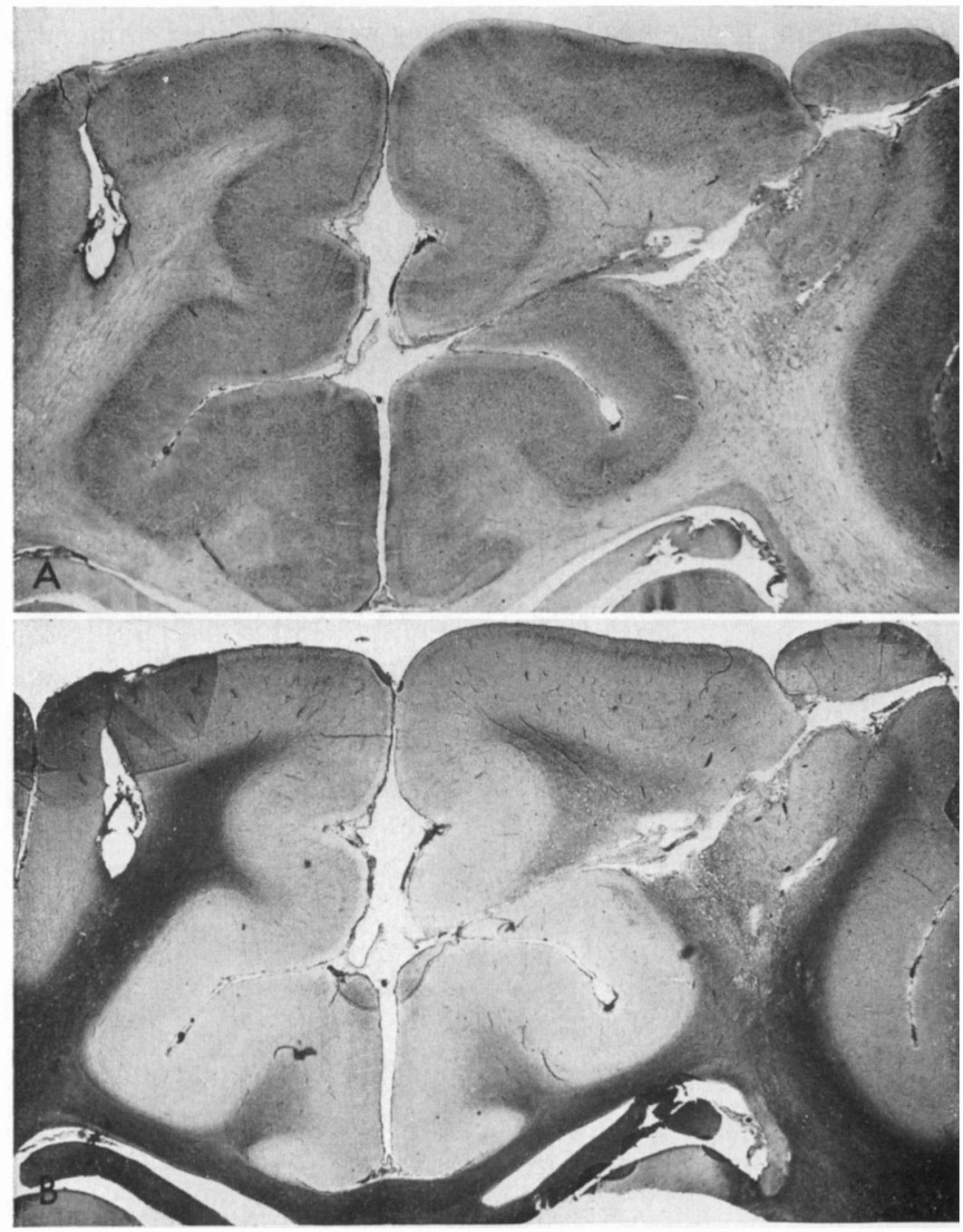

Fig. 3. Histological sections from cat 55-46, killed 1 month after "isolations." Electrodes were $3.5 \mathrm{~mm}$ from midline; undercut $15 \mathrm{~mm}$ long, $5 \mathrm{~mm}$ to posterior or anterior egress. Preoperative threshold for U-cut side $0.4 \mathrm{ma}$, postoperative $0.25 \mathrm{ma}$ with immediate effectiveness. Undercut side required 200 trials of retraining; $0.7 \mathrm{ma}$ preoperative threshold, $0.7 \mathrm{ma}$ postoperative after retraining. A, Nissl; B, Weil stain; $8 \times$. 


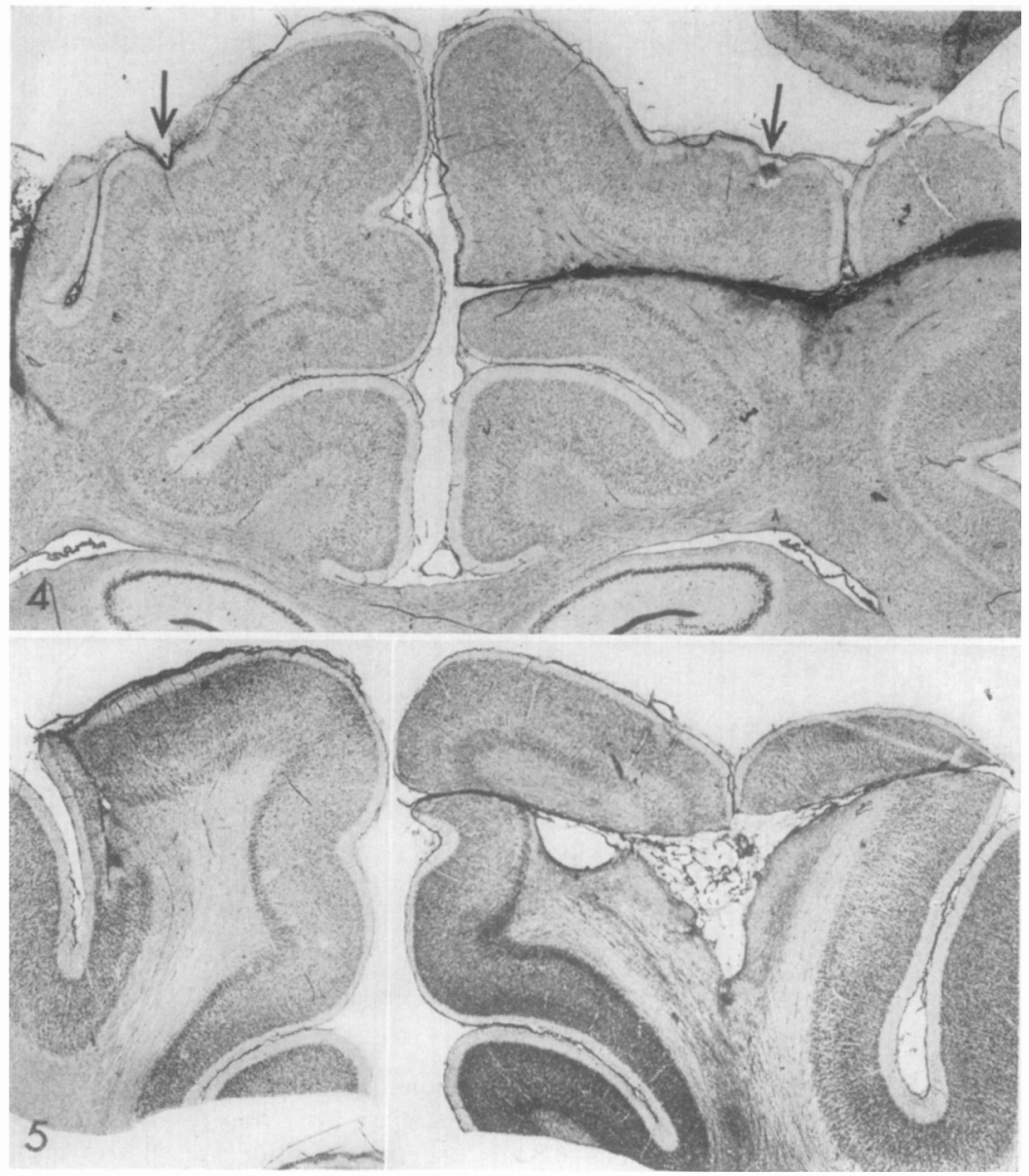

Fig. 4. Histological section from cat 58-14, killed 2 months after "isolations." Arrows point to two electrode locations; U-cut farther laterally than on most. Electrodes $4-5 \mathrm{~mm}$ lateral to midline. Undercut $11 \mathrm{~mm}$ long, $4 \mathrm{~mm}$ to posterior or anterior egress. Preoperative threshold for U-cut side $0.9 \mathrm{ma}, 0.8 \mathrm{ma}$ postoperative. Undercut side required 360 trials of retraining; $0.3 \mathrm{ma}$ preoperative threshold, 1.0 ma postoperative after retraining. Nissl stain; $8 \times$.

Fic. 5. Histological sections from cat 55-51, killed 4 months after "isolations." Sections are from different levels. Electrodes were 4-5 $\mathrm{mm}$ lateral to midline. Undercut $12 \mathrm{~mm}$ long, $4.5 \mathrm{~mm}$ to posterior or anterior egress. Undercut side required 380 trials retraining; $0.4 \mathrm{ma}$ preoperative threshold, $0.8 \mathrm{ma}$ postoperative after retraining. Nissl stain; $8 \times$. 
stable response level. Current thresholds for CR elicitation were determined before and after the surgical procedures using a constant current stimulus converter.

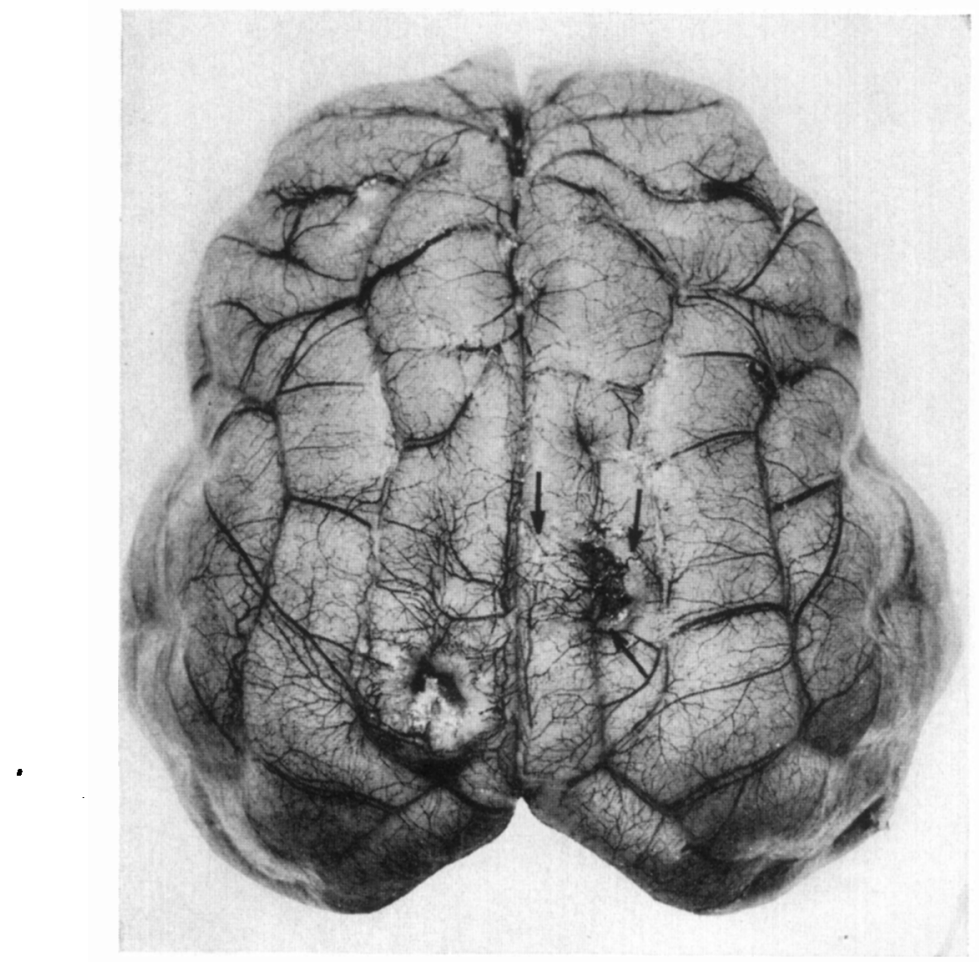

Fig. 6. Pial vascular pattern revealed by India ink perfusion one month after U-cut and complete circumsection on right and left marginal gyri, respectively. Cuts went through pia to depth of about $4 \mathrm{~mm}$. Living tissue absent inside scarred area on left although minimal vascular invasion into scar can be seen. On right, arrows point to blood vessels which have grown across and into scarred area. Regrowth occurred from pial flap which can be seen to be heavily vascularized. One end of U-shaped cut extended just medial to blood vessel at most medial arrow, cortex subpially cut from this point to midline. On posterior tip of $\mathrm{U}$, subpial cut ended slightly more laterally leaving large vessel there intact.

\section{Results}

Postisolation Performance. Tests on the ten animals with U-shaped cuts and undercuts of stimulated areas were begun no longer than 1 week after surgery. For the initial tests an equal number of trials (five to ten CS-US pairs) was usually given for each hemisphere at CS intensities considerably 
above preoperative CS thresholds. Foot-flexion CR were counted as before, and when performance to stimulation of the $U$-cut side reached about $40 \%$ CR (42\% average for the group; Table 1), training was usually concentrated on the other, undercut cortex.

For comparison of postoperative performances, Table 1 shows the level of $\mathrm{CR}$ to stimulation of both hemispheres for a given number of trials in each of the ten animals. For example, cat 55-27 gave seven $C R$ to

TABLE 1

Postoperative Response Level and Retraining Trials Necessary to Reach Criterion for the Ten Bilaterally Isolated Animals

\begin{tabular}{|c|c|c|c|c|c|c|c|c|}
\hline \multirow[b]{3}{*}{ Cat } & \multicolumn{4}{|c|}{ Postisolation performance ${ }^{a}$} & \multicolumn{4}{|c|}{ Retraining } \\
\hline & \multicolumn{2}{|c|}{ Undercut } & \multicolumn{2}{|c|}{ U-cut } & \multicolumn{2}{|c|}{ Undercut $b$} & \multicolumn{2}{|c|}{$\mathrm{U}$-cut ${ }^{b}$} \\
\hline & $(\%)$ & (Trials) & $(\%)$ & (Trials) & $(\%)$ & (Trials) & $(\%)$ & (Trials) \\
\hline $55-27$ & 0 & 35 & 20 & 35 & 60 & 324 & 60 & 200 \\
\hline $55-46$ & 0 & 15 & 67 & 15 & 64 & 204 & 64 & 12 \\
\hline $55-51$ & 0 & 15 & 47 & 15 & 53 & 350 & 53 & 30 \\
\hline $56-5$ & 20 & 15 & 100 & 15 & 64 & 400 & 100 & 15 \\
\hline $58-6$ & 26 & 35 & 37 & 35 & 70 & 235 & 70 & 85 \\
\hline $58-14$ & 0 & 50 & 65 & 50 & 65 & 360 & 65 & 50 \\
\hline 58-18 & 0 & 20 & 35 & 20 & 65 & 200 & 65 & 60 \\
\hline $59-6$ & 0 & 40 & 45 & 40 & 45 & 375 & 45 & 40. \\
\hline $59-16$ & 0 & 20 & 65 & 20 & 25 & 650 & 25 & 8 \\
\hline $59-18$ & 0 & 50 & 4 & 50 & 70 & 277 & 70 & 305 \\
\hline M & 4.6 & & 48.5 & & & 337.5 & & 80.5 \\
\hline $\mathbf{M}(\text { data })^{c}$ & 4 & & 42 & & & & & \\
\hline
\end{tabular}

a Distinction between this and "retraining" arbitrary. Postoperative tests all accomplished in 5 days or less.

$b$ Taken for highest level of response obtained for stimulation of undercut cortex.

c Means determined from number of responses rather than from averaged percentage.

thirty-five presentations (20\%) of CS to U-cut cortex, but no CR in. thirty-five stimulations of undercut cortex. It is obvious from Table 1 that with two exceptions, 56-5 and 58-6, CR in these initial tests were obtained only from stimulation of U-cut cortex. However, since these were correlated comparisons (16) and the performance level and number of trials varied, a test was made for the significance of the distribution of differences between the levels of performance for the two conditions, undercutting versus $U$ cutting, when these differences were greater than 
zero. That the immediate difference in postoperative performance is significantly greater than zero can be judged from the $t$-ratio of 4.22 and the probability $(P)$, with nine degrees of freedom, between 0.01 and 0.001 .

Postisolation Retraining. Postoperative retraining was continued with $\mathrm{CS}$ to both undercut and $\mathrm{U}$-cut areas until a comparable and consistent level of $C R$ was reached. Complete retraining to stimulation of the undercut cortex was often difficult so that the ultimate level of performance of 25 to $70 \%$ for this group was below the preoperative level of 60 to $100 \%$. A statistical comparison was possible between retraining trials as shown in Table 1 for undercut versus U-cut cortex when a given criterion of CR level had been reached. Again the results were overwhelmingly in favor of the U-cut cortex; but note that one cat, 59-18, required prolonged retraining with stimulation of U-cut cortex. The $t$-ratio of 4.47 had a $P$, with nine degrees of freedom, between 0.01 and 0.001 . Reliable CR current thresholds were obtained on seventeen of the twenty cortical sites. Thresholds for the undercut areas averaged $100 \%$ higher than preoperative thresholds, whereas thresholds for the U-cut cortex were only $21 \%$ higher. Considering the experimental conditions and the method of determining thresholds (7), these differences are thought to be valid and to support the interpretation that relatively less disturbance to $C R$ results from the U-cutting.

The individual data for retraining provide some interesting comparisons. As might be expected, there is much variability. For example, cat 59-6, with bilateral trigeminal neurotomy prior to electrode implantation, reached a $45 \%$ CR level to stimulation of the $\mathrm{U}$-cut area in forty trials, whereas 375 trials were required for it to reach the same level to stimulation of the undercut area. In the original training 520 trials were required to reach criterion where an auditory $\mathrm{CS}$ was used but on the first day transfer to cortical CS at the marginal gyrus locations (subsequently undercut and U-cut) was immediate. This cat's record also can be considered a good control over any disruptive process which might be attributed to the surgical procedures per se or to the possible effects of prolonged recovery time. On the third postoperative day stimulation of the U-cut cortex produced CR with no particular difficulty, but none was produced by stimulation of the undercut area even at $2.0 \mathrm{ma}$. The animal was then set aside for 1 month without further training. Upon resumption of training, stimulation of the U-cut area immediately elicited CR, but 350 trials were necessary before stimulation of the undercut area again became effective. Thresholds for the undercut side were $0.25 \mathrm{ma}$ pre- 
operatively, 1.0 ma postoperatively (but $3 \mathrm{sec} \mathrm{CS}$ ), and for the U-cut side 0.1 ma preoperatively, 0.4 ma postoperatively.

Another example of the marked differences between undercut and U-cut cortex can be observed in the data on cat 58-6 (Table 1 and Fig 2). Initially this cat reached comparable levels of $\mathrm{CR}$ performance to $\mathrm{CS}$ in this order: photic, 360 trials; left marginal gyrus (later U-cut), 105 trials, threshold $0.3 \mathrm{ma}$; and right marginal gyrus (later undercut), ninety trials, threshold $0.3 \mathrm{ma}$. After surgical isolation, on the first test day, the CR level was: photic, five CR out of ten trials; U-cut, five of ten and undercut three of five, using $0.7 \mathrm{ma}$ for the cortical CS in each case. Although the levels of performance for stimulation at the undercut and U-cut areas on the first day seemed similar, it soon became apparent that $\mathrm{CR}$ to stimulation of the undercut cortex were extremely unstable and erratic. No reliable and consistent performance was recorded until after 230 trials had been given. A 70\% CR level and a threshold of 0.7 ma were finally obtained. Only eighty-five postoperative trials had been required to achieve a stable $70 \%$ level for stimulation of the U-cut area, and its threshold was $0.5 \mathrm{ma}$. There was no posterior egress from the undercut zone and the nearest electrode was $3.5 \mathrm{~mm}$ from the midline and $5 \mathrm{~mm}$ from the anterior egress.

Bilateral Undercuts. Four of the fourteen cats were studied following bilateral undercuts of the cortical CS areas so that such things as difference in reacquisition of $\mathrm{CR}$ between a long (anterior-posterior) cut and a shorter one, transfer from a "retrained" side to the other side, and threshold changes could be studied.

Not surprisingly, and similarly to the cases of unilateral undercutting, the length of cut makes a difference in ease of $C R$ reacquisition; the shorter the cut, the more quickly $C R$ reappear and the shorter the retraining to a stable CR level. Thus one important factor appears to be the amount of cortex through which excitation must be elaborated or conducted to be effective.

A posterior surgical approach bilaterally undercutting the suprasylvian gyri was used in one cat. One side was quickly retrained after a $10-\mathrm{mm}$ undercut, but in more than 700 trials the opposite gyrus never again mediated a CS to give a CR. On this refractory side the cut extended far into the anterior pole for more than $20 \mathrm{~mm}$. Nonundercut cortex was about $9 \mathrm{~mm}$ from the stimulating electrodes in the latter case and $4 \mathrm{~mm}$ in the former.

Data from two of the bilaterally undercut cats are in contrast with 
data from previous work showing transfer or generalization within a modality and between hemispheres (9), and suggest distinctly that a retrained undercut side cannot produce this type of general responsiveness. The animal must be completely retrained to respond to stimulation of the homotopic undercut area.

It appears to make no difference for the right foreleg $\mathrm{CR}$ on which side the undercut is made, but the data are scanty and this conclusion cannot be considered final.

Trigeminal Neurotomies and Histology. Meningeal stimulation near the midline can produce $\mathrm{CR}$ but this possible source of artifact can be eliminated by trigeminal neurotomy (7). Bilateral neurotomies were successful on three of the four cats with bilaterally undercut cortex, and on four of the other ten animals. In the latter instance neurotomies were performed on three animals prior to any training. Two other cats were unilaterally neurotomized on the undercut side. Only one cat, omitted from this series, gave evidence of trigeminal afferent stimulation by the CS. Otherwise, with the exception of two animals obtunded by additional brain stem damage, the neurotomized animals were similar to the others in thresholds, retraining requirements and general performance for stimulation of either undercut of U-cut sides.

Stimulating current spread to "intact" tissue can also lead to spurious results. A CS of 0.7 ma was capable of stimulating "intact" tissue when the electrodes were within $1 \mathrm{~mm}$ of a border of a U-type cut. In this case CR persisted after neurotomy and after total isolation produced by undercutting the area. With electrodes $2 \mathrm{~mm}$ from a cut border, the CS must exceed 1.0 ma before there is danger of effective current spread to "intact" tissue.

Our histological study confirms that of Clark and Sunderland (5), who found almost no degeneration of cortical elements after section of the white matter and in our material this holds even when the white matter is totally destroyed. The sections showed several successful attempts to further isolate an undercut area by extending the cut vertically to the surface at the posterior or anterior extent. For the marginal gyrus at least, either a posterior or an anterior projection can provide effective egress.

For some undercut cortex a $3-\mathrm{sec} \mathrm{CS}$ is more effective than a 2 -sec one. One neurotomized cat, with only anterior egress $5 \mathrm{~mm}$ from the electrodes on the undercut area, had an ultimate threshold of $0.2 \mathrm{ma}$ for the longer pulse train and $1.0 \mathrm{ma}$ for the shorter. 


\section{Discussion}

The results show unequivocally that subcortical rather than transcortical projections are the most important for effective elaboration of a cortically applied electrical CS. This directly contradicts the similar, but poorly controlled, experiments of Kogan (14); and lends no support to the Pavlovian concepts of "irradiation" and intracortical elaboration of CS-US connections. It is not assumed in the present experiments that normal neuronal activity is initiated by the electrical CS, nor can antidromic be distinguished from orthodromic effects. The results are nevertheless concordant with those data indicating that some cortical systems are organized via vertical (radial) rather than horizontal (tangential) connections. Sperry and his colleagues $(21,22)$ have shown that multiple division of the cortical network by a grid of subpial sections does not impair motor or visual function. This demonstrates, as do the present experiments, albeit with a more "unnatural" input, that horizontal organization is nonessential. A pattern of columnar organization is also present at the microscopic level for both somatosensory (17) and visual (12) cortex.

The supposition of the primacy of the vertical organization does not deny the possibility of significant intracortical connections. Experiments of Lashley (15) and Chow (4) in fact show that behaviorally useful neural transactions can pass via surviving intracortical pathways. The retraining data of the present experiments confirm this fact, but with additional peculiarities.

In view of the great readiness with which trained cats respond to a new CS (9), a certain mystery attaches to the failure of stimulation of the undercut areas to produce CR consistently without complete retraining. It is possible that some specific reorganization of cortical conduction or elaboration is required and occurs only as a consequence of training. The fact that animals with short intracortical conduction paths to "intact" cortex relearn faster than do those with longer undercuts, lends this suggestion moderate support. The most important alternative is that the undercut cortex conducts the excitation adequately from the first test day, and the retraining required is for "recognition" of this perhaps weak or exceedingly bizarre signal.

The surface-negative cortical response to directly applied electrical stimuli, as studied by Adrian (1) and others (e.g., 2, 18) could unquestionably propagate the necessary $5 \mathrm{~mm}$ to gain egress from the undercut areas. Little is known of the corticifugal capabilities of this direct (super- 
ficial) cortical response. Under Adrian's (1) conditions it was the deep cortical response and not the superficial that occurred with effective stimulation of "motor" cortex. The direct cortical response is propagated largely by $U$ fibers and readily follows the 50 -cycle/sec stimulus used here as CS (18). It is likely that the tetanic CS also sets up the slower burst response described by Burns (3).

Further evidence that electrical stimulation of undercut cortex produces propagated effects is given by Kogan (13). Although this report also suffers from lack of histological details, he found that stimulation of undercut sensorimotor cortex still affected the excitability of visual cortex. Both Kogan (13) and Burns (3), as well as others, have shown that undercut cortex is influenced in its electrical activity by adjacent, "intact" cortex. It thus seems likely that electrical action can be immediately transmitted in and out of an undercut cortical area.

It is conceivable that the excitability of the appropriate synaptic systems has been reduced by the "deafferentation" and consequent "disuse" (10), and that repetition of the CS alone might have a restorative effect. Although this possibility deserves study, it will be complicated by the concomitant extinction achieved by isolated application of the CS; and there is nothing in Grafstein and Sastry's (11) data on chronically isolated cortex to suggest a significant reduction of excitability. Fiber regrowth, as described by Rose, Malis and Baker (19), or supersensitivity presumably would occur independently of training, and it was shown here that the mere passage of time did not see a return of CS effectiveness. Electrophysiological analysis of undercut cortex in this behavioral context may, however, provide important information on modifications of neural conduction directly relates to a learned association.

\section{References}

1. Adrian, E. D. 1937. The spread of activity of the cerebral cortex. J. Physiol. London 88: 127-161.

2. Brooks, V. B., and P. S. ENGER. 1959. Spread of directly evoked responses in the cat's cerebral cortex. J. Gen. Physiol. 42: 761-777.

3. Burns, B. D. 1958. "The Mammalian Cerebral Cortex." Arnold Publ., London.

4. CHow, K. L. 1961. Anatomical and electrographical analysis of temporal neocortex in relation to visual discrimination learning in monkeys, pp. 507-525. In: "Brain Mechanisms and Learning." A. Fessard, R. W. Gerard, J. Konorski, and J. F. Delafresnaye [eds.], Blackwell Scientific Publications, Oxford.

5. Clark, W. E. Le Gros, and S. Sunderland. 1939. Structural changes in the isolated visual cortex. J. Anat. 73: 563-574. 
6. Doty, R. W. 1961. Conditioned reflexes formed and evoked by brain stimulation, pp. 397-412. In: "Electrical Stimulation of the Brain." D. E. Sheer [ed.], Univ. of Texas Press, Austin.

7. Doty, R. W., L. T. Rutiedge, and R. M. Larsen. 1956. Conditioned reflexes established to electrical stimulation of cat cerebral cortex. J. Neurophysiol. 19: 401-415.

8. Doty, R. W., and L. T. Rutiedge. 1959. Conditioned reflexes elicited by stimulation of partially isolated cerebral cortex. Federation Proc. 18: 37.

9. Doty, R. W., and L. T. RuTLEDGE. 1959. "Generalization" between cortically and peripherally applied stimuli eliciting conditioned reflexes. J. Neurophysiol. 22: $428-435$.

10. Eccles, J. C., and A. K. McIntyre. 1953. The effects of disuse and of activity on mammalian spinal reflexes. J. Physiol. London 121: 492-516.

11. Grafstein, B., and P. B. Sastry. 1957. Some preliminary electrophysiological studies on chronic neuronally isolated cerebral cortex. Electroencephalog. and Clin. Neurophysiol. 9: 723-725.

12. Hubes, D. H., and T. N. WiEser. 1962. Receptive fields, binocular interaction and functional architecture in cat's visual cortex. J. Physiol. London 160: 106154.

13. Kogan, A. B. 1960. The manifestation of processes of higher nervous activity in the electrical potentials of the cortex during free behavior of animals, pp. 51-64. In: "The Moscow Colloquium on Electroencephalography of Higher Nervous Activity." H. H. Jasper and G. D. Smirnov [eds.]. Electroencephalog. and Clin. Neurophysiol. (Suppl. 13).

14. Kogan, A. B. 1961. On the structure of the closing mechanism of the conditioned reflex. Zhur. Vysshę Nervnoi Deyatel'nosti im I.P. Pavlova 9: 651659. [In Russian.]

15. LASHIEY, K. S. 1943. Studies of cerebral function in learning. XII, Loss of the maze habit after occipital lesions in blind rats. J. Comp. Neurol. 79: 431-462.

16. MCNemar, Q. 1959. "Psychological Statistics." Wiley, New York.

17. MountcastLE, V. B. 1957. Modality and topographic properties of single neurons of cat's somatic sensory cortex. $J$. Neurophysiol. 20: 408-434.

18. OCHS, S., and H. BookER. 1961. Spatial and temporal interaction of direct cortical responses. Exptl. Neurol. 4: 70-82.

19. Rose, J. E., L. I. Matis, and C. P. Baker. 1961. Neural growth in the cerebral cortex after lesions produced by monoenergetic deuterons, pp. 279-301. In: "Sensory Communication." W. A. Rosenblith [ed.], Wiley, New York.

20. Rutledge, L. T., and R. W. Doty. 1956. Neural pathways of conditioned reflexes to cortical stimulation. Federation Proc. 15: 158.

21. Sperry, R. W. 1947. Cerebral regulation of motor coordination in monkeys following multiple transection of sensorimotor cortex. J. Neurophysiol. 10: 275-294.

22. Sperry, R. W., N. Miner, and R. E. Myers. 1955. Visual pattern perception following subpial slicing and tantalum wire implantations in the visual cortex. J. Comp. Physiol. Psychol. 48: 50-58.

23. SunderLand, S. 1938. The production of cortical lesions by devascularization of cortical areas. J. Anat. 73: 120-129. 